\title{
The influence of lymph node status on the overall survival in surgically treated hilar cholangiocarcinoma
}

\author{
Nicolae BACALBASA ${ }^{1,2,3}$, Irina BALESCU ${ }^{4}$, Mihaela VILCU ${ }^{2,3}$, Iulian BREZEAN ${ }^{2,3}$ \\ ${ }^{1}$ Center of Excellence in Translational Medicine, Fundeni Clinical Institute, Bucharest, Romania \\ 2"Carol Davila" University of Medicine and Pharmacy, Bucharest, Romania \\ "Ion Cantacuzino" Clinical Hospital, Bucharest, Romania \\ ${ }^{4}$ Ponderas Academic Hospital, Bucharest, Romania
}

\begin{abstract}
Cholangiocarcinoma represents an extremely aggressive digestive malignancy associated with very poor survival rates in the absence of a treatment with curative intent. Moreover, an important per cent of patients are diagnosed in advanced stages of the disease, when resection with curative intent is no longer feasible. When it comes to cases which can be submitted to surgery, radical resection, including lymph node dissection, seems to be one of the most important therapeutic strategies in order to improve survival. This is a literature review of the most relevant studies which were focused on analyzing the impact of lymph node status on the overall survival of surgically treated hilar cholangiocarcinomas.
\end{abstract}

Keywords: hilar cholangiocarcinoma, resection, lymph node dissection

\section{INTRODUCTION}

Cholangiocarcinoma refers to a tumoral subtype arising from the epithelial cells lining in the biliary ducts on the whole length of the intrahepatic and extrahepatic ducts, but excluding the ampulla of Vater and the gall bladder $(1,2)$. The overall prognostic of these lesions remains extremely poor, with estimated five year survival rates of $5 \%$ and $17 \%$ for intrahepatic and extrahepatic malignant tumors respectively (3).

The role of radical surgery in patients with hilar cholangiocarcinomas

Among these cases, hilar cholangiocarcinoma accounts for up to $70 \%$ of cases and is associated with poor prognostic due to multiple rea- sons: it has an aggressive biological behavior, associated with a significant chemoresistance, with high risk of invading the surrounding vascular structures and becoming unresectable (3). Therefore, only a small proportion of patients are candidates for surgery; even so, the mortality rate ranges between 5 and $10 \%$. In the last decades, due to the improvement of the surgical techniques and of the perioperative management of these patients, even more aggressive surgical procedures have been proposed in order to improve the long term outcomes of them (4).

In such cases, surgery with curative intent should comprehend extended hepatic resection en bloc with biliary resection and lymph node dissection; however, in certain cases, due to the 
presence of venous invasion, portal vein resection might be also associated in order to achieve a radical surgical procedure, with negative resection margins $(5,6)$. When it comes to the criteria of unresectable disease, according to Zaydfudim, resection should be not performed in cases presenting with distal metastases, lymph node metastases beyond the hepatoduodenal ligament, bilateral extension at the level of the segmental biliary branches, and by the occlusion of the portal vein or common hepatic artery proximal to the bifurcation (7).

When it comes to the extent of the lymph node dissection and the impact of the number of the retrieved nodes on the staging of patients with central cholangiocarcinomas, it seems that at least seven lymph nodes should be harvested in order to provide an adequate staging and prognostic, while harvesting more than 15 nodes seem not to influence anymore the staging and the prognostic of such cases (8).

\section{The impact of lymph node metastases after resection with curative intent for hilar cholangiocarcinomas}

Lymph node status, including the status of the para-aortic lymph nodes represents one of the most important prognostic factors among patients with central cholangiocarcinomas; however, routine lymph node dissection is not widely performed, this surgical procedure being reported in 3-53\% of cases (9-11). As expected, it seems that most often an extended lymph node dissection is carried out in the Eastern countries; however, in Eastern patients the diagnostic is usually established more rapidly, the tumors being usually less extended when compared to the Western patients. These differences are clearly demonstrated in the paper conducted by Kimura et al. and published in 2017 (12). In this article the authors compared the outcomes of the patients diagnosed with hilar cholangiocarcinoma who were submitted to surgery in an Eastern hospital (Hirosaki University Hospital, Japan) to those submitted to surgery in an Western hospital (St. James University Hospital, Leeds, United Kingdom) between January 1995 and December 2014. During this period 80 patients were submitted to surgery in the Eastern hospital, their outcomes being compared to a similar group of 103 patients submitted to surgery in the Western hospital. There was no significant difference between the two groups in regard of sex distribution, associated comorbidities or in regard to the necessity of as- sociating a preoperative biliary drainage; interestingly, an important difference was encountered in terms of age at diagnostic, Eastern patients being significantly older when compared to the Western counterparts. As for the extent of the surgical approach, the Western patients were usually diagnosed in more advanced stages of the disease, the proportion of cases necessitating more extended hepatic resections, as well as the association of vascular or other visceral resections being higher. Although cases submitted to surgery in the Western hospital were in more advanced stages of disease, no significant difference was encountered between the two groups in regard to the extent of the lymph node dissection or in regard to the number of metastatic lymph nodes. Moreover, an RO resection was more frequently encountered in the Eastern countries. In univariate analysis, the number of positive lymph nodes was associated with a poorer outcome in the both cohorts (12).

Although it can be suspected that the number of retrieved nodes is proportional to the extent of the surgical procedure, it seems that this fact is not always true; in a study conducted by Xiong et al. and published in 2015 the authors included 52 patients submitted to surgery for Bismuth types I and II hilar cholangiocarcinoma, and demonstrated that the rates of the harvested lymph nodes were similar between cases submitted solely to bile duct resection, when compared to those submitted to bile duct resection and hepatic resection; moreover, it seems that the number of metastatic nodes was similar between the two categories (13).

One of the most important factors after radical resection of hilar cholangiocarcinomas is represented by the status of the retrieved lymph nodes; therefore in a Japanese study conducted in Nagoya and published in 2005 (4) the authors included 301 patients submitted to surgery with curative intent and demonstrated that patients presenting lymph node metastases experienced an overall survival significantly poorer when compared to those in whom the lymphatic nodules were negative. However, the authors underlined the fact that the presence of micrometastatic disease did not influence the overall survival in cases with otherwise negative nodes (4). Another interesting observation of this study was the one that patients submitted to lymph node dissection had a better overall survival, even if they presented metastatic nodes, when compared to unresected cases (4). 
Another interesting subject is the one related to the impact of the number of harvested lymph nodes on the overall survival in patients with central cholangiocarcinoma. According to Gugliemi's study, the median overall survival seems to be significantly influenced by this parameter (14). Therefore, in the study conducted by this author on a group of 62 patients submitted to surgery with curative intent for hilar cholangiocarcinomas, cases in which more than three lymph nodes were harvested reported a significantly improved overall survival when compared to those in which 1 to 3 nodes had been harvested or to those in which lymph node dissection was not performed $(p<0.01)$. Moreover, the authors demonstrated that the overall survival was not influenced by the station in which metastatic disease had been found (N1 versus $\mathrm{N} 2$ versus $\mathrm{N} 3, p=0.66$ ). In multivariate analysis the authors demonstrated that the radicality of resection as well as the lymph node ratio were the only significant prognostic factors for the long term outcomes (14).

In order to offer a better chance to receive a tailored treatment after resection and to improve the long term outcomes of these patients, the Chinese authors created a formula in which they introduced the number of lymph node metastases, the serum levels of CEA and the surgical margins, these three parameters being the most significant ones in multivariate analysis for survival (15). These parameters were quoted with 1 point if the resection margin was positive, if the lymph nodes presented metastases and if the serum level of CEA was higher than 15 $\mathrm{ng} / \mathrm{ml}$, and 2 points if the resection margin was negative, if the lymph nodes did not present metastatic disease and if the serum levels were lower than $15 \mathrm{ng} / \mathrm{ml}$. According to the value of this score, the 169 cases introduced in this study were classified in three categories - high risk, middle risk and low risk, the three categories reporting a significant difference in terms of survival (15).
Another aspect which was intensively studied in patients with hilar cholangiocarcinomas is related to the influence of lymph node micrometastases on the overall survival. In the study conducted by Taniguchi et al. and published in 2006 (16) the authors included 28 patients submitted to surgery for hilar cholangiocarcinomas in whom the histopathological studies demonstrated the absence of lymph node metastases; totally, the authors harvested 423 regional lymph nodes which were immunostained with antibodies against cytokeratines 8 and 18. After performing this method the authors reported the presence of micrometastatic deposits in 11 of the 28 patients (corresponding to 14 of the 423 harvested lymph nodes). In univariate analysis the presence of micrometastatic disease was significantly correlated to the tumoral stage at diagnostic, with the histopathological degree of differentiation, as well as with the presence of venous invasion; moreover, micrometastatic disease significantly affected the overall survival rate; therefore the five year overall survival rate was $21.8 \%$ in cases with micrometastatic disease and $66.5 \%$ in cases with no micrometastatic deposits $(p=0.02)$. Therefore, the authors concluded that immunohistochemical studies should be routinely performed among patients with negative nodes in order to identify the subset of cases presenting micrometastatic lesions (16).

\section{CONCLUSIONS}

Hilar cholangiocarcinomas remain an aggressive subtype of digestive malignancies associated with poor rates of survival in the absence of radical surgical treatment. In order to improve the outcomes of these patients and to provide an adequate staging of the tumoral process, regional lymph node dissection is mandatory. Moreover, certain authors proposed stratifying the cases after calculating a risk score which might further assure a tailored treatment.

Conflict of interest: none declared Financial support: none declared

\section{REFERENCES}

1. Callea F, Sergi C, Fabbretti G et al. Precancerous lesions of the biliary tree. J.Surg. Oncol.Suppl 1993; 3: 131-3.

2. Murakami Y, Uemura K, Sudo T et al. Adjuvant gemcitabine plus S-1 chemotherapy improves survival after aggressive surgical resection for advanced biliary carcinoma. Ann.Surg. 2009; 250: 950-6.

3. Squadroni M, Tondulli L, Gatta G et al. Cholangiocarcinoma. Crit Rev. Oncol. Hematol. 2017; 116: 11-31.

4. Nishio $H$, Nagino $M$, Nimura Y. Surgical management of hilar cholangiocarcinoma: the Nagoya experience. HPB (Oxford) 2005; 7: 259-62.

5. Nagino M, Ebata T, Yokoyama $Y$ et al. Evolution of surgical treatment for perihilar cholangiocarcinoma: a single-center 34-year review of 574 consecutive resections. Ann.Surg. 2013; 258: 129-40. 
6. Nagorney DM, Kendrick ML. Hepatic resection in the treatment of hilar cholangiocarcinoma. Adv.Surg. 2006; 40: 159-71.

7. Zaydfudim VM, Rosen CB, Nagorney DM. Hilar cholangiocarcinoma. Surg. Oncol. Clin.N.Am. 2014; 23: 247-63.

8. Kambakamba P, Linecker M, Slankamenac $\mathrm{K}$ et al. Lymph node dissection in resectable perihilar cholangiocarcinoma: a systematic review. Am.J.Surg. 2015; 210: 694-701.

9. Kosuge T, Yamamoto J, Shimada K et al. Improved surgical results for hilar cholangiocarcinoma with procedures including major hepatic resection. Ann. Surg. 1999; 230: 663-71.
10. Nimura Y, Hayakawa N, Kamiya J et al. Hepatic segmentectomy with caudate lobe resection for bile duct carcinoma of the hepatic hilus. World J.Surg. 1990; 14: 535-43.

11. Neuhaus P, Jonas S, Bechstein WO et al. Extended resections for hilar cholangiocarcinoma. Ann.Surg. 1999; 230: 808-18.

12. Kimura N, Young AL, Toyoki $Y$ et al. Radical operation for hilar cholangiocarcinoma in comparable Eastern and Western centers: Outcome analysis and prognostic factors. Surgery 2017; 162: 500-14.

13. Xiong J, Nunes QM, Huang W et al. Major hepatectomy in Bismuth types I and II hilar cholangiocarcinoma. J.Surg.Res. 2015; 194: 194-201.

14. Guglielmi A, Ruzzenente A, Campagnaro T et al. Prognostic significance of lymph node ratio after resection of peri-hilar cholangiocarcinoma. HPB (Oxford) 2011; 13: $240-5$.

15. Wang Y, Duan B, Yan L et al . Long-term outcome after surgical resection for cholangiocarcinoma and prognostic index value. Surgeon. 2016; 14: 38-43.

16. Taniguchi $K$, Tabata M, lida T et al. Significance of lymph node micrometastasis in pNO hilar bile duct carcinoma. Eur.J.Surg. Oncol. 2006; 32: 208-12. 